Case Report Journal of Epilepsy Research pISSN 2233-6249 / eISSN 2233-625

Received January 21, 2018

Accepted June 25, 2018

Corresponding author: Ok Joon Kim, MD, PhD Department of Neurology, CHA Bundang Medical Center, CHA University, 59 Yatap-ro, Bundang-gu, Seongnam 13496, Korea

Tel. +82-31-780-5481

Fax. $+82-31-780-5269$

E-mail; okjun77@cha.ac.kr

\section{Tonic Upward Eyeball Deviation Mimicking Non-Convulsive Occipital Lobe Status Epilepticus That Was Induced by Hydrocephalus}

\author{
Min-Hee Woo, MD, Jung-Won Shin, MD, PhD, Seung-Hun Oh, MD, PhD, Ok Joon Kim, MD, PhD \\ Department of Neurology, CHA Bundang Medical Center, CHA University, Seongnam, Korea
}

\begin{abstract}
Several seizure-like symptoms are difficult to differentiate from epileptic convulsion, and then if they were misdiagnosed, they could be led to grave prognosis. A 41-year-old man was referred to the emergency room due to unconsciousness. Brain computed tomography (CT) revealed acute subdural hemorrhage along the left frontal lobe, and intraparenchymal hemorrhage in the left temporo-occipital lobe. After emergent decompressive craniectomy, he recovered an alert mental state but became progressively drowsy. Four days later, virtually continuous tonic upward eyeball deviation was observed. He had been taking antiepileptic drugs following an occipital lobectomy 20 years prior due to intractable epilepsy, and we assumed these upward eyeball deviations were symptoms of non-convulsive occipital lobe status epilepticus. Hence, doses and classes of antiepileptic drugs were modified, but clinical manifestations did not improve. Follow-up brain CT revealed newly developed hydrocephalus and compression of the mesencephalon. His symptoms fully resolved after a ventriculo-peritoneal shunt operation. In this case report, we describe the case of a patient exhibiting tonic upward eyeball deviation induced by hydrocephalus that was not associated with a seizure. (2018;8:49-53)
\end{abstract}

Key words: Hydrocephalus, Non-convulsive status epilepticus, Occipital lobe epilepsy, Tonic upgaze

\section{Introduction}

Many clinical conditions mimic seizure, including syncope, hypoglycemia, hemi-facial spasm, non-epileptic myoclonus, transient ischemic attack, and panic disorder. ${ }^{1}$ Sometimes these conditions are difficult to differentiate from epileptic convulsion, and they can easily lead to misdiagnoses. In cases of non-convulsive status epilepticus (NCSE), diagnosis is even more challenging because symptoms and signs are variable, subtle, and ill-defined. ${ }^{2}$ Electroencephalography (EEG) is also nonspecific in many cases. Misdiagnosis can lead to serious problems including undue delays in appropriate treatment. Herein, we describe the case of a patient with symptoms mimicking non-convulsive occipital lobe status epilepticus that were associated with hydrocephalus after traumatic hemorrhage.

\section{Case}

A 41-year-old man collapsed on the street and was found unconscious. He had a history of left occipital lobectomy at the age of
22 years due to intractable occipital lobe epilepsy (OLE). He was taking multiple antiepileptic drugs (AEDs) including levetiracetam 2,000 mg/day, valproic acid $500 \mathrm{mg} / \mathrm{day}$, and carbamazepine 200 $\mathrm{mg} /$ day. Previous brain computed tomography (CT) revealed encephalomalacia of the left parieto-occipital lobe (Fig. 1A), and EEG revealed focal slowing in the left centro-parieto-temporal region (Fig. 2A). Despite having undergone occipital lobectomy, he had more than 5 recurrent seizure episodes within a year. In the emergency room, brain CT revealed acute subdural hemorrhage in the left frontal lobe and intraparenchymal hemorrhage in the left temporo-occipital lobe (Fig. 1B). Emergent decompressive craniectomy was performed to remove the subdural hemorrhage, but the intraparenchymal hemorrhage in the left temporo-occipital lobe remained. After the surgery, he regained consciousness and was able to perform wheelchair ambulation. The Glasgow coma scale (GCS) score at this point was 14 (eye 4, verbal 4, motor 6). However, 4 days after the surgery he became less responsive and had frequent episodes of unconsciousness accompanied by tonic upward deviations of the eyeballs lasting a few seconds. The GCS score was 9 (eye 2, verbal 3, motor 4) and abrupt 

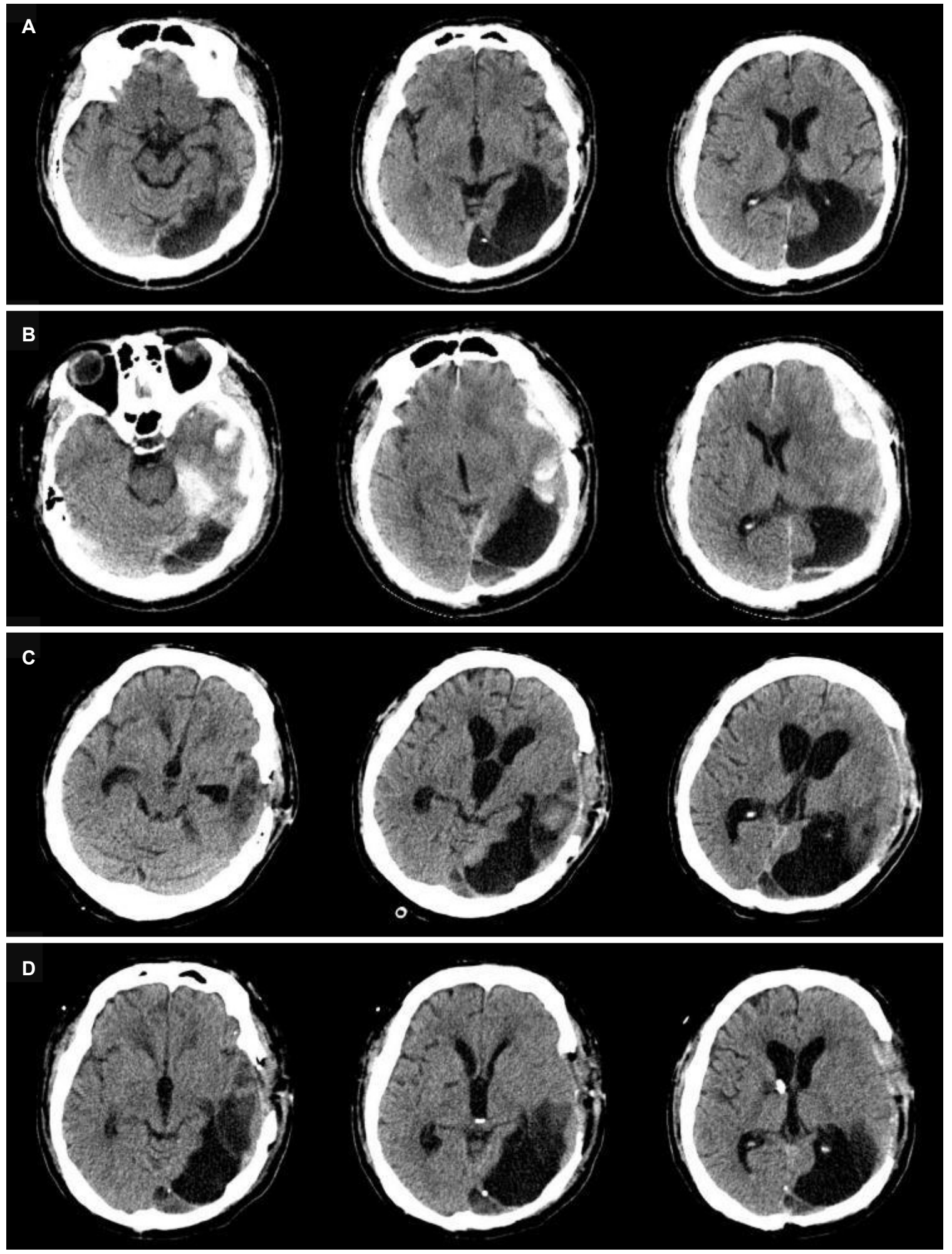

Figure 1. Serial brain computed tomography. Encephalomalacia of the left parieto-occipital lobe due to occipital lobectomy for intractable epilepsy 4 months before admission (A). Acute subdural hemorrhage in the left frontal lobe and intraparenchymal hemorrhage in the left temporo-occipital lobe were detected in the emergency room (B). A substantial amount of intraparenchymal hemorrhage remained in the left temporo-occipital lobe. Bilateral enlargement of the lateral ventricles, temporal horns, and third ventricle and compression of the midbrain were observed, suggesting obstructive hydrocephalus (C). Four weeks after a shunt operation, hydrocephalus was improved (D). 
mental drowsiness continued for more than 30 minutes. We initially considered this condition to be a result of non-convulsive occipital lobe status epilepticus, because he was being treated for OLE and there was an associated high possibility of exacerbation due to hemorrhage. Scalp EEG revealed frequent spikes or sharp waves in the left temporo-parietal lobe, and occasional frontal spikes and sharp waves suggesting ongoing seizure caused by hemorrhage (Fig. 2B). Intravenous lorazepam was injected at the time of the epi- sodes, and simultaneously a modified AED regimen was administered consisting of levetiracetam from 2,000 mg/day to $3,000 \mathrm{mg} /$ day, valproic acid from $500 \mathrm{mg} /$ day to $1,000 \mathrm{mg} /$ day, phenytoin from 200 $\mathrm{mg} /$ day to $300 \mathrm{mg} /$ day, lamotrigine $400 \mathrm{mg} /$ day, lacosamide 100 $\mathrm{mg} /$ day, and carbamazepine $400 \mathrm{mg} /$ day. Although scalp EEG ceased to depict spikes and sharp waves (Fig. 2C), his GCS score worsened to 7 (eye 2, verbal 2, motor 3) and he exhibited virtually continuous tonic upward eyeball deviation.

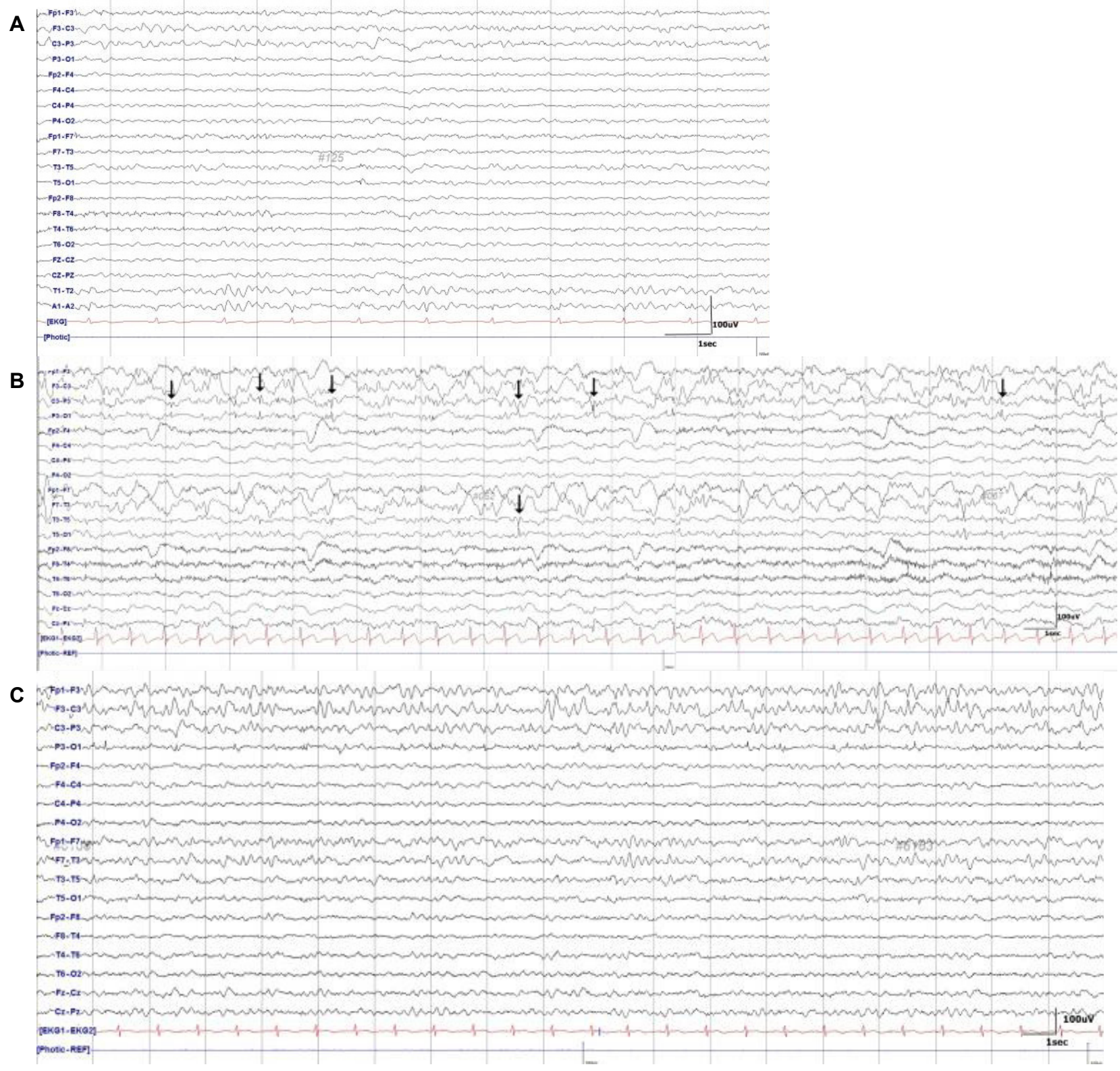

Figure 2. Focal slowing in the left centro-parieto-temporal region was evident on baseline scalp electroencephalography 2 months prior to admission (A). Disorganized background activity with rhythmic delta slowing superimposed on frequent spikes or sharp waves at the left temporo-occipital lobe were evident during symptoms of tonic upward gaze eyeball deviation. The arrows indicate spikes or sharp waves (B). After modification of the antiepileptic drug regimen, alpha waves were evident without spikes or sharp waves (C). 
Because there was no clinical improvement, further evaluation was necessary to investigate alternative causes of tonic upward eyeball deviation and mental state. Follow-up brain CT revealed bilateral enlargement of lateral ventricles, temporal horns, and the third ventricle suggesting obstructive hydrocephalus (Fig. 1C) and associated midbrain compression. To alleviate hydrocephalus, external ventricular drainage was performed followed by a ventriculo-peritoneal shunt operation (Fig. 1D). Tonic upward eyeball deviation ceased to occur, and the patient recovered consciousness. The GCS score at this point was 13 (eye 3, verbal 4, motor 6). Thereafter, doses of AEDs were reduced and adjusted to levetiracetam 1,000 mg/day, phenobarbital 60 $\mathrm{mg} /$ day, phenytoin $400 \mathrm{mg} /$ day, and lamotrigine $400 \mathrm{mg} /$ day. The patient was transferred to the rehabilitation department for active rehabilitation, and there were no recurrences of tonic upward eyeball deviation.

\section{Discussion}

In cases of NCSE, symptoms can range from mild confusion to coma. ${ }^{3}$ Thus, it is important to distinguish NCSE from other pathological conditions that causea confused mental state. Unconscious patients hospitalized in intensive care units due to head injury or other medical conditions are often not diagnosed with NCSE due to a lack of definite visible ictal symptoms. ${ }^{4}$ It is also very difficult to differentiate between the progression of underlying disease and ineffective treatment of NCSE in cases of combined serious medical or surgical conditions. Recently, we encountered an impending case mimicking non-convulsive occipital lobe status epilepticus that was induced by hydrocephalus after intracranial hemorrhage.

The typical symptoms of OLE are visual auras, visual hallucinations, ictal blindness, eye movement sensations, blinking, and eyelid fluttering. ${ }^{5}$ Paroxysmal tonic deviation of the eyeball is also acharacteristic symptom of OLE. Ictal manifestations of OLE depend on the spread of epileptic discharge, and diagnosing it can be challenging because well-localized unifocal rhythmic ictal discharge is infrequent and rapid ictal spread to other brain areas. ${ }^{5}$ Surface ictal scalp EEG in patients with OLE often reveals posterior temporal activity and diffuse posterior sharp waves or spike activity with a wide field of distribution. ${ }^{6}$ The most common etiologic factor of OLE is prenatal or neoplastic lesion, and head trauma is the second most common. ${ }^{7}$

In the present case, the patient exhibited tonic upward eyeball deviations in conjunction with unconsciousness. Initially our diagnosis was non-convulsive occipital lobe status epilepticus, because he had a previous history of recurrent seizures after occipital lobectomy, and he had also recently developed an intracranial hemorrhage-a condition that has the potential to increase seizure activity. EEG improvement without clinical improvement was concordant with a possible diagnosis of NCSE according to the most recent International League Against Epilepsy description and classification of NCSE. ${ }^{8}$ Based on the results of a series of scalp EEGs and follow-up brain CTs, we surmised that the tonic upward eyeball deviation originated from structural lesion rather than epilepsy. The mesodiencephalic junction is the critical site of upward gaze control, because the anatomical structures mediating vertical-gaze input to the oculomotor nuclei are all clustered around the Sylvian aqueduct. ${ }^{9}$ Any structural lesions directly or indirectly irritating this area including stroke, vasculitis, or tumor could cause involuntary eyeball movement. We could not completely rule out the possibility of NSCE, which may have developed due to irritation of mesencephalic areas by chemical components that emerged after intracranial hemorrhage. However, it is reasonable to conclude that tonic upward eyeball deviation was induced by compression of mesodiencephalic brain due to hydrocephalus development after intracranial hemorrhage. Fortunately, via emergent surgery we were able to prevent serious conditions such as brain herniation and death.

In conclusion, tonic upward eyeball deviation may occur in non-convulsive occipital lobe status epilepticus patients, but structural lesions may induce similar signs and symptoms. Clinicians should be aware of seizure-mimicking medical or surgical conditions that are not true seizures, especially in NCSE patients, to prevent grave prognoses.

\section{Acknowledgements}

This study was partly supported by a grant of the Korea Healthcare Technology R\&D Project (H116C1559), Ministry for Health, Welfare \& Family Affairs, Republic of Korea.

\section{Conflicts of Interest}

The authors declare that they have no financial or other conflicts of interest in relation to this research and its publication.

\section{References}

1. Benbadis S. The differential diagnosis of epilepsy: a critical review. 
Epilepsy Behav 2009;15:15-21.

2. Laccheo I, Sonmezturk $H$, Bhatt $A B$, et al. Non-convulsive status epilepticus and non-convulsive seizures in neurological ICU patients. Neurocrit Care 2015;22:202-11.

3. Pollak L, Gandelman-Marton R, Margolin N, Boxer M, Blatt I. Clinical and electroencephalographic findings in acutely ill adults with non-convulsive vs convulsive status epilepticus. Acta Neurol Scand 2014; 129:405-11.

4. Sutter R, SemmlackS, Kaplan PW. Nonconvulsive status epilepticus in adults-insights into the invisible. Nat Rev Neurol 2016;12:281-93.

5. Yang PF, Jia YZ, Lin Q, et al. Intractable occipital lobe epilepsy: clin- ical characteristics, surgical treatment, and a systematic review of the literature. Acta neurochir (Wien) 2015;157:63-75.

6. Taylor I, Scheffer IE, Berkovic SF. Occipital epilepsies: identification of specific and newly recognized syndromes. Brain 2003;126:753-69.

7. Kuzniecky R. Symptomatic occipital lobe epilepsy. Epilepsia 1998;39 Suppl 4:S24-31.

8. Beniczky S, Hirsch $\amalg$, Kaplan PW, et al. Unified EEG terminology and criteria for nonconvulsive status epilepticus. Epilepsia 2013;54 Suppl 6:28-9.

9. Moncayo J, Bogousslavsky J. Oculomotor disorders in vertebrobasilar stroke. Expert Rev Ophthalmol 2009;4:259-81. 
